\title{
Development of an Algorithm \\ for Defining Thyristors Opening Angle Value \\ at the New Configuration of Grid Commutation \\ of Reversible Converter Arms of Electric Locomotive
}

\author{
Oleg V. Chikirkin ${ }^{a}$, Oleg V. Melnichenko, \\ Aleksey O. Linkov ${ }^{\mathrm{b}}$ and Sergey G. Shramko ${ }^{\mathrm{b}}$ \\ ${ }^{a}$ Traction Directorate, the branch of JSCo «RZD» \\ Moscow, Russian Federation \\ ${ }^{b}$ Irkutsk State Transport University \\ Irkutsk, Russian Federation
}

\begin{abstract}
The article presents a new approach in the application of a diode discharge arm which is aimed at increasing the energy indicators of traction mode of AC locomotives. The method implies the utilization of electromagnetic energy stored in the commutate current circuit for closing exhausted thyristor arms of traction converters through a buffer contour provided by the discharge arm.

A mathematical simulation of electromagnetic processes of the standard and proposed converters has been performed. The analysis of the results allows to find the most optimal value of thyristor arms opening angle when the discharge arm closes fully the exhausted thyristor arms in compliance with the condition of increasing the power factor of the electric locomotive.

The developed control method of the proposed converter is presented by a table of pulses fed to the thyristors electrodes, as well as by the algorithm of point-of-time calculation of the impulses generation.
\end{abstract}

Keywords: AC locomotives, converter equipment, electromagnetic processes, commutation, control algorithms, power factor.

Acknowledgements. The study was carried out within the framework of the state assignment for state work No. AAAA-A20-121050600025-2 dated 05/06/2021. 


\title{
Разработка алгоритма определения величины
}

\section{угла открытия тиристоров при новой организации \\ сетевой коммутации плеч выпрямительно-инверторного \\ преобразователя электровоза}

\author{
О.В. Чикиркин ${ }^{\mathrm{a}}$, О.В. Мельниченко \\ А. О. Линьков ${ }^{\sigma}$, С.Г. Шрамко \\ а Дирекичи тяги - филиал ОАО «РЖД» (ЦТ) \\ Российская Федерация, Москва \\ ${ }^{\sigma}$ Иркутский государственный университет путей сообщения \\ Российская Федерачия, Иркутск
}

\begin{abstract}
Аннотация. В статье предложен прогрессивный подход в применении разрядного диодного плеча с целью повышения энергетических показателей тягового режима электровозов переменного тока. Способ подразумевает использование электромагнитной энергии, запасенной в цепи выпрямленного тока, для закрытия отработавших тиристорных плеч тяговых преобразователей через буферный контур, обеспечиваемый разрядным плечом.

Проведено математическое моделирование электромагнитных процессов работы штатного и предлагаемого преобразователей. Анализ результатов позволяет обнаружить наиболее оптимальную величину угла открытия тиристорных плеч, при котором разрядное плечо полностью закрывает отработавшие плечи с соблюдением условия повышения коэффициента мощности электровоза.

Разработанный способ управления предлагаемого преобразователя представлен таблицей подаваемых на электроды тиристоров импульсов, а также алгоритмом вычисления момента времени формирования этих импульсов.
\end{abstract}

Ключевые слова: электровозы переменного тока, преобразовательная техника, электромагнитные процессы, коммутация, алгоритмы управления, коэффициент мощности.

Благодарности. Исследование выполнено в рамках государственного задания по государственной работе № AАAА-А20-121050600025-2 от 06.05.2021.

Цитирование: Чикиркин, О.В. Разработка алгоритма определения величины угла открытия тиристоров при новой организации сетевой коммутации плеч выпрямительно-инверторного преобразователя электровоза / О.В. Чикиркин, О. В. Мельниченко, А. О. Линьков, С. Г. Шрамко // Журн. Сиб. федер. ун-та. Техника и технологии, 2021, 14(8). С. 903-913. DOI: $10.17516 / 1999-494 X-0361$

\section{Введение}

В начале 2018 г. Новочеркасским электровозостроительным заводом (НЭВЗ) был построен электровоз 3ЭС5К «Ермак» № 879 с опытными тяговыми выпрямительно-инверторными преобразователями серии ВИП-4000Д (изготовитель АО «Электровыпрямитель», г. Саранск), в которых применено разработанное в 2002-2012 гг. учеными ИрГУПС и ДВГУПС техническое решение, заключающееся в использовании дополнительного разрядного диодного плеча, присоединенного анодом к минусовой шине тягового преобразователя, а катодом - к плюсовой [1-3].

Выпуск НЭВЗ опытного электровоза серии «Ермак» говорит о том, что данное техническое решение является одним из перспективных направлений совершенствования тягового электропривода с коллекторными тяговыми электродвигателями (ТЭД). Однако такое решение требует 
дальнейших исследований по повышению энергетических показателей электровозов переменного тока, составной частью которых выступает разработка новых алгоритмов, связанных с организацией коммутации тиристорных плеч.

Проведенные исследования показали, что для выпрямительно-инверторных преобразователей (ВИП) с разрядным диодным плечом наилучшие результаты по энергетическим характеристикам электровоза получаются при применении двухконтурного алгоритма с одновременной коммутацией $[4,5]$. Так, при анализе электромагнитных процессов, полученных при моделировании на математической модели «тяговая подстанция - контактная сеть - электровоз» в имитационной среде Simulink для режима тяги электровоза переменного тока [6, 7], авторами был сделан вывод, что применение алгоритма двухконтурной одновременной коммутации позволяет осуществлять закрытие предыдущих работающих плеч ВИП. Это возможно, если до определенного предела увеличивать минимальный угол открытия тиристорных плеч преобразователя. В свою очередь, накопленная электромагнитная энергия в цепи выпрямленного тока будет разряжаться через буферный контур благодаря разрядному диодному плечу, что может привести к повышению коэффициента мощности и устойчивости работы электровоза. При таком техническом решении с целью максимального использования накопленной энергии в цепи выпрямленного тока авторами предлагается увеличить минимальный угол открытия $\alpha_{0}$ тиристорных плеч ВИП $[8,9]$.

При применении штатной (типовой) схемы ВИП без разрядного плеча направление работ по повышению коэффициента мощности электровоза было связано только с уменьшением угла $\alpha_{0}$ и практически сразу себя исчерпало, так как снизить минимальный угол $\alpha_{0}$ ниже 9 электрических градусов (далее - ${ }^{\circ}$ эл.) не представляется возможным в силу угрозы нарушения потенциальных условий работы плеч ВИП, что приводит к возникновению бросков тока на ТЭД электровоза и других аварийных режимов [10-12]. Увеличение же угла $\alpha_{0}$ сразу вызывало резкое повышение генерации электровозом реактивной мощности при эксплуатации, пусть при этом и наблюдалась более устойчивая его работа. Последнее не могли не заметить наладчики электронных систем электровозов, поэтому минимальный угол открытия тиристорных плеч ВИП $\alpha_{0}$ на практике можно встретить с величиной до $18^{\circ}$ эл. (при соблюдении достаточных потенциальных условий на анодных шинах преобразователя и при $9^{\circ}$ эл.). Это, в свою очередь, приводит к снижению технической скорости электровоза и пропускной способности тягового участка, степень которого предстоит оценить в дальнейших работах.

\section{Результаты математического моделирования}

В табл. 1 приведены результаты эксперимента оценки коэффициента мощности электровоза в зависимости от длительности угла открытия $\alpha_{0}$, полученные при моделировании работы электровоза в режиме тяги на примере середины 4-й зоны (далее - зона 3,5 ) регулирования напряжения электровоза в двух случаях: штатная силовая схема и алгоритм управления ВИП, а также схема с разрядным диодным плечом и новым алгоритмом управления.

Из данных табл. 1 видно, что с увеличением угла открытия тиристоров плеч ВИП $\alpha_{0}$ при работе штатного ВИП коэффициент мощности электровоза резко падает. Предлагаемый же алгоритм управления ВИП с разрядным диодным плечом при аналогичных нагрузках увеличивает коэффициент мощности электровоза в среднем на 3,3 \% относительно штатного. Это объясняется уменьшением времени коммутации плеч VS7 и VS8 преобразователя за счет сни-

$$
-905-
$$


Таблица 1. Результаты исследования работы ВИП при различных минимальных углах открытия тиристорных плеч $\alpha_{0}$ для зоны 3,5 регулирования напряжения при штатной и предлагаемой схемах

Table 1. Results of converter operation research with varying minimal firing angles $\alpha_{0}$ of its thyristor arms at zone 3.5 of voltage regulation for standard and proposed circuits

\begin{tabular}{|c|c|c|c|c|c|c|c|c|}
\hline \multirow{2}{*}{$\begin{array}{c}\text { Параметр } \\
\text { Угол } \alpha_{0},{ }^{\circ} \text { эл. }\end{array}$} & \multicolumn{8}{|c|}{ Значение } \\
\hline & 5 & 9 & 15 & 20 & 26 & 27 & 28 & 29 \\
\hline \multicolumn{9}{|c|}{ Штатная силовая схема } \\
\hline Коэффициент мощности $\chi$, отн. ед. & 0,773 & 0,765 & 0,747 & 0,727 & 0,695 & 0,689 & 0,683 & 0,676 \\
\hline \multicolumn{9}{|c|}{ Предлагаемая силовая схема } \\
\hline Амплитуда тока разрядного диодного плеча, А & 600 & 800 & 1000 & 1250 & 1600 & 1610 & 1610 & 1610 \\
\hline Коэффициент мощности $\chi$, отн. ед. & 0,78 & 0,781 & 0,783 & 0,786 & 0,791 & 0,787 & 0,778 & 0,707 \\
\hline
\end{tabular}

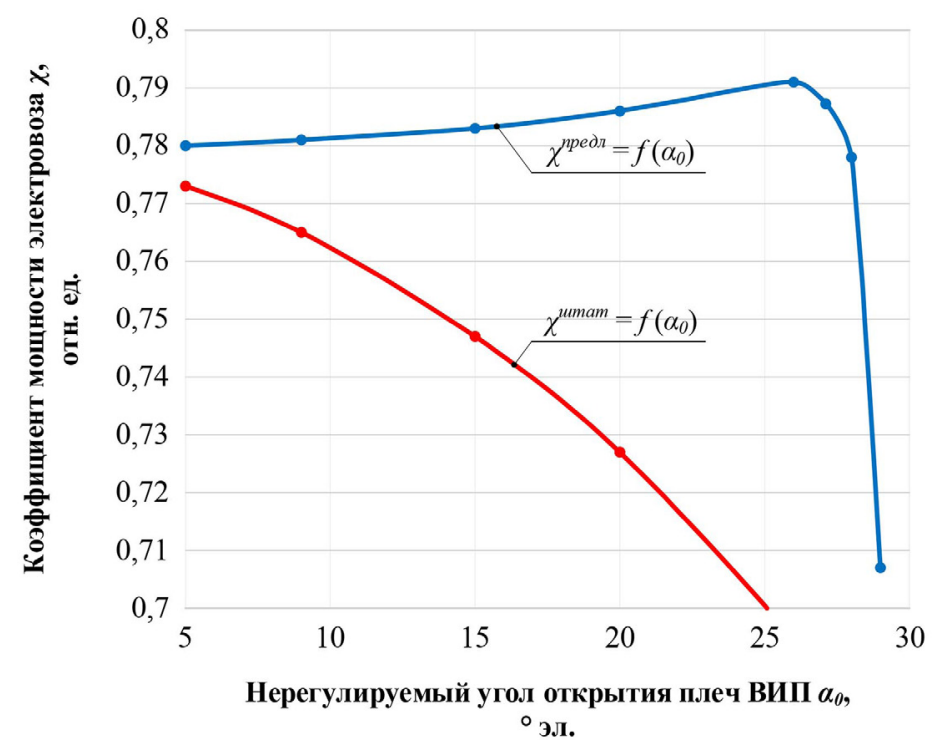

Рис. 1. Зависимость коэффициента мощности $\chi$ электровоза от длительности минимального угла открытия тиристоров $\alpha_{0}$ для зоны 3,5 регулирования напряжения при штатной и предлагаемой силовых схемах

Fig. 1. Dependance between electric locomotive power factor $\chi$ and the length of thyristor minimal firing angle $\alpha_{0}$ at zone 3.5 of voltage regulation for standard and proposed power circuits

жения протекающего через них коммутационного тока, так как часть накопленной энергии в цепи выпрямленного тока замыкается через разрядное диодное плечо.

По данным табл. 1 построены зависимости коэффициента мощности $\chi$ электровоза от минимального угла открытия $\alpha_{0}$ при различных схемах ВИП и способах их управления (рис. 1). Видно, что при минимальном угле открытия $\alpha_{0}=26^{\circ}$ эл. электровоз имеет наибольший коэффициент мощности.

Такое увеличение коэффициента мощности электровоза объясняется полученными при моделировании электромагнитными процессами ВИП. На рис. 2-4 изображены диаграммы токов плеч многозонного выпрямителя для двухконтурного алгоритма с одновременной ком-

$$
-906-
$$




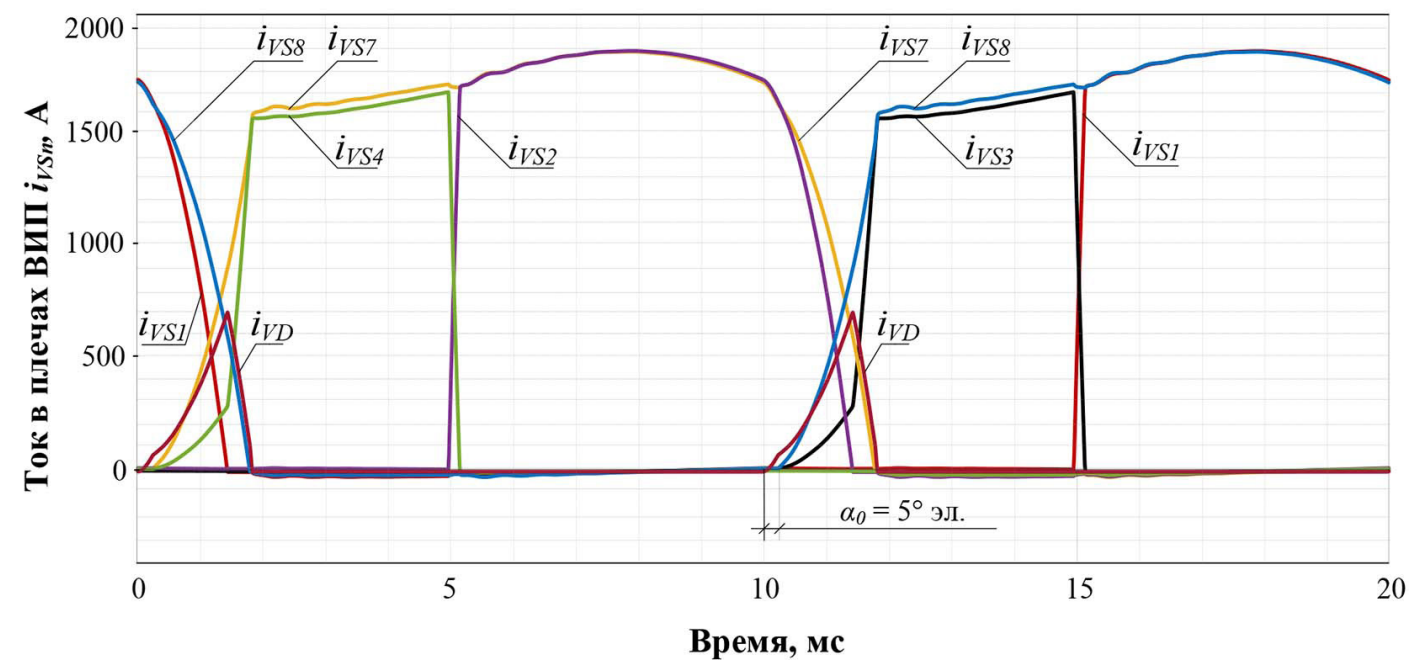

Рис. 2. Диаграммы токов плеч многозонного выпрямителя с разрядным диодным плечом и одновременной коммутацией тиристоров ВИП при работе на зоне 3,5 регулирования напряжения с углом $\alpha_{0}=5^{\circ}$ эл.

Fig. 2. Diagrams of arm currents of a multizone rectifier with a diode discharge arm and simultaneous commutation of converter thyristors at zone 3.5 of voltage regulation with $\alpha_{0}=5$ electrical degrees

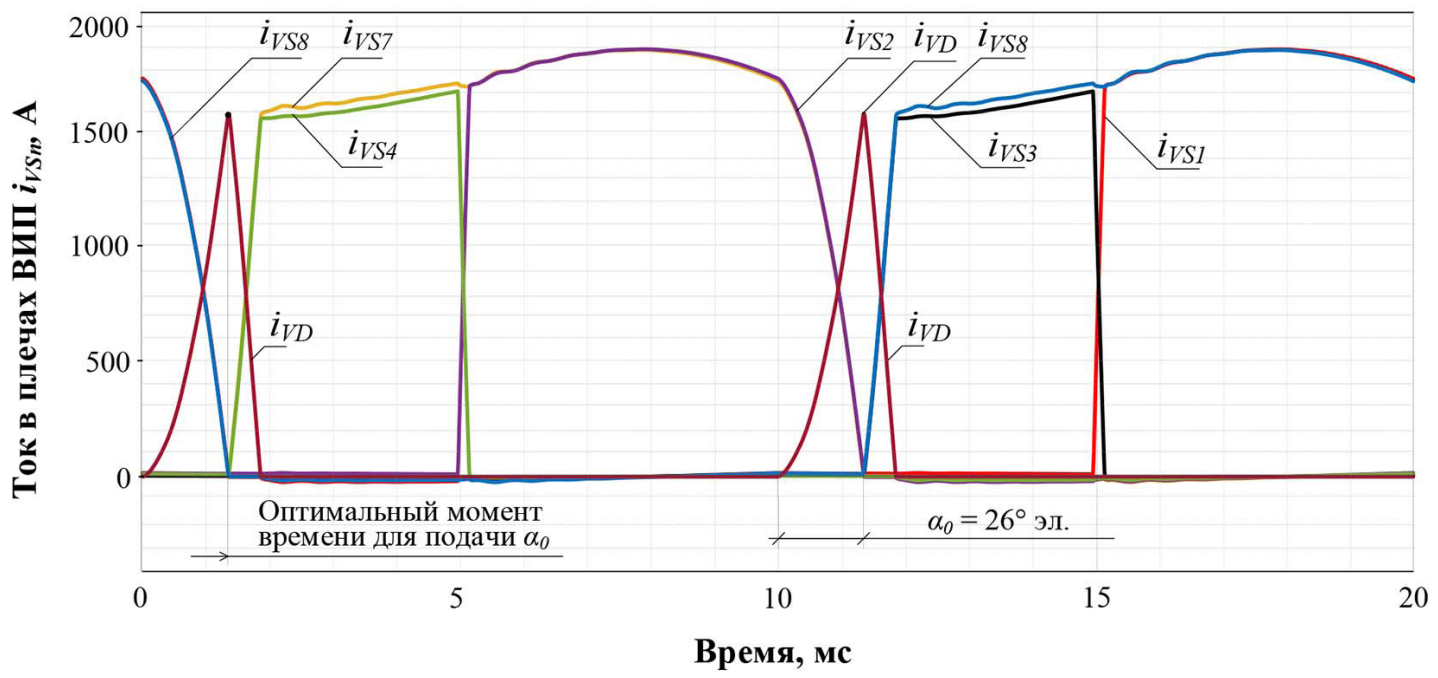

Рис. 3. Диаграммы токов плеч многозонного выпрямителя с разрядным диодным плечом и одновременной коммутацией тиристоров ВИП при работе на зоне 3,5 регулирования напряжения с углом $\alpha_{0}=26^{\circ}$ эл.

Fig. 3. Diagrams of arm currents of a multizone rectifier with a diode discharge arm and simultaneous commutation of converter thyristors at zone 3.5 of voltage regulation with $\alpha_{0}=26$ electrical degrees

мутацией тиристорных плеч ВИП при работе на зоне 3,5 регулирования напряжения с различными по длительности углами открытия: $\alpha_{0}=5^{\circ}$ эл., $\alpha_{0}=26^{\circ}$ эл. и $\alpha_{0}=29^{\circ}$ эл.

На представленных диаграммах электромагнитных процессов видно, что при малых значениях угла $\alpha_{0}$ ток через диод $i_{V D}$ не успевает достигнуть максимального значения (мгновенного значения тока ТЭД) и, соответственно, энергия, накопленная в цепи выпрямленного тока, используется нагрузкой не полностью (рис. 2). Закрытие предыдущих (отработавших) плеч

$$
-907-
$$




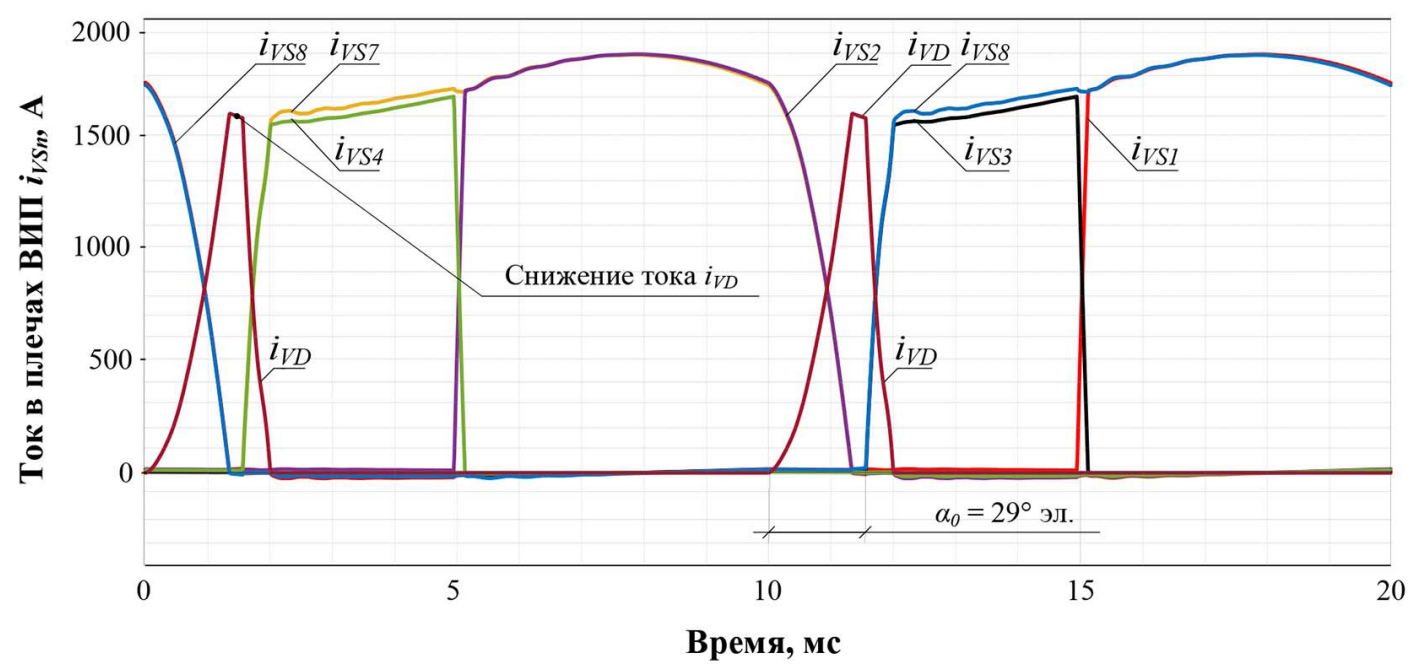

Рис. 4. Диаграммы токов плеч многозонного выпрямителя с разрядным диодным плечом и одновременной коммутацией тиристоров ВИП при работе на зоне 3,5 регулирования напряжения с углом $\alpha_{0}=29^{\circ}$ эл.

Fig. 4. Diagrams of arm currents of a multizone rectifier with a diode discharge arm and simultaneous commutation of converter thyristors at zone 3.5 of voltage regulation with $\alpha_{0}=29$ electrical degrees

ВИП VS1 и VS8 обеспечивается открытием последующих (вступающих в работу) плеч VS4 и VS7, что опять приводит к классической коммутации плеч с короткозамкнутым контуром в плечах преобразователя, а это затягивает процесс коммутации.

В случае же больших углов $\alpha_{0}$ ток через диод $i_{V D}$ достигает своего максимума и начинает снижаться, что показано на кривой $i_{V D}$ (рис. 4). При этом наблюдается резкое снижение коэффициента мощности, как демонстрирует рис. 1. Следовательно, угол $\alpha_{0}$ необходимо выбирать таким образом, чтобы ток через разрядное плечо $i_{V D}$ достигал своего непосредственного максимума (рис. 3). Такой способ организации коммутации полностью отличается от классического способа. Происходит перераспределение нагрузки по плечам, причем разрядное диодное плечо за счет накопленной электромагнитной энергии в цепи выпрямленного тока выполняет полезную работу (закрытие плеч, например, VS1 и VS8 или VS2 и VS7) и значительно сокращает время короткого замыкания в плечах преобразователя, что снижает коммутационные потери.

Анализ электромагнитных процессов и данных табл. 1 показывает, что максимальное значение $\chi$ достигается при подаче нерегулируемого угла $\alpha_{0}$ в момент времени, соответствующий максимальному току через разрядное плечо до момента начала его спада (рис. 4). Для заданных условий моделирования оптимальный угол $\alpha_{0}$ получился равным $26^{\circ}$ эл.

\section{Анализ полученных результатов}

Очевидно, что максимальное значение тока через разрядное плечо зависит от тока тяговых двигателей, который, в свою очередь, меняется в процессе регулирования силы тяги электровоза. Принимая это во внимание, авторы предлагают изменять угол $\alpha_{0}$ пропорционально изменению тока ТЭД, при этом коэффициент мощности электровоза будет постоянно достигать максимально возможного значения. При такой организации коммутации меняется назначение угла $\alpha_{0}$, который теперь не имеет минимально допустимого значения, а становится управляю- 
щим импульсом для перевода тиристоров плеч ВИП из закрытого состояния в открытое. Тогда новый алгоритм управления плечами многозонного выпрямителя с учетом одновременной коммутации тиристорных плеч ВИП приобретает вид, представленный в табл. 2.

Предлагаемый алгоритм имеет ряд достоинств перед штатным, используемым в настоящее время на современных отечественных электровозах переменного тока:

- на 1-й зоне регулирования отсутствует необходимость в формировании минимального угла открытия $\alpha_{0}$, что упрощает и повышает надежность системы управления плечами ВИП, поскольку теперь накопленная электромагнитная энергия в цепи выпрямленного тока разряжается в каждом полупериоде через буферный контур, образованный разрядным диодным плечом;

- на последующих зонах регулирования (2-4) отсутствует необходимость в формировании дополнительного задержанного импульса управления с фазой $\alpha_{03}$, благодаря чему снижается длительность коммутации и, следовательно, увеличивается средневыпрямленное напряжение на тяговых двигателях;

- на зонах регулирования 2-4 диодное плечо в начале каждого полупериода напряжения создает буферный контур разряда электромагнитной энергии, которая способна надежно закрывать отработавшие тиристорные плечи, тем самым совершая полезную работу;

- алгоритм реализуется при упрощении силовой схемы электровоза, поскольку исключаются трансформаторы напряжения (датчики потенциальных условий).

Чтобы максимально использовать энергию, накопленную в цепи выпрямленного тока, необходимо определять момент времени, в который ток через разрядное диодное плечо достигает своего максимума. Одновременно это условие будет являться моментом подачи импульсов управления на последующие плечи ВИП, вступающие в работу в данном полупериоде напряжения.

Для реализации предлагаемого технического решения следует установить в цепь разрядного диодного плеча датчик тока, выходной сигнал которого будет подаваться в штатную си-

Таблица 2. Новый алгоритм управления ВИП с разрядным диодным плечом в режиме тяги электровоза Table 2. New reversible power converter control algorithm with a diode discharge arm in electric locomotive's traction mode

\begin{tabular}{|c|c|c|c|c|c|c|c|c|c|}
\hline \multirow{2}{*}{$\begin{array}{c}\text { Зона } \\
\text { регулирования }\end{array}$} & \multirow{2}{*}{$\begin{array}{c}\text { Направление } \\
\text { ЭДС } \\
\text { (полупериод) }\end{array}$} & \multicolumn{8}{|c|}{ Импульсы управления на плечи ВИП } \\
\hline & & VS1 & VS2 & VS3 & VS4 & VS5 & VS6 & VS7 & VS8 \\
\hline \multirow{2}{*}{1} & $\leftarrow$ & - & - & $\alpha_{p}$ & - & - & $\alpha_{p}$ & - & - \\
\hline & $\rightarrow$ & - & - & - & $\alpha_{p}$ & $\alpha_{p}$ & - & - & - \\
\hline \multirow{2}{*}{2} & $\leftarrow$ & $\alpha_{p}$ & - & $\alpha_{0}$ & - & - & $\alpha_{0}$ & - & - \\
\hline & $\rightarrow$ & - & $\alpha_{p}$ & - & $\alpha_{0}$ & $\alpha_{0}$ & - & - & - \\
\hline \multirow{2}{*}{3} & $\leftarrow$ & - & - & $\alpha_{p}$ & - & $\alpha_{0}$ & - & - & $\alpha_{0}$ \\
\hline & $\rightarrow$ & - & - & - & $\alpha_{p}$ & - & $\alpha_{0}$ & $\alpha_{0}$ & - \\
\hline \multirow{2}{*}{4} & $\leftarrow$ & $\alpha_{p}$ & - & $\alpha_{0}$ & - & - & - & - & $\alpha_{0}$ \\
\hline & $\rightarrow$ & - & $\alpha_{p}$ & - & $\alpha_{0}$ & - & - & $\alpha_{0}$ & - \\
\hline
\end{tabular}


стему управления МСУД-Н, и доработать программное обеспечение согласно разработанному ниже алгоритму (рис. 5).

Для определения момента включения плеч, вступающих в работу, измеряется ток через разрядное диодное плечо с частотой дискретизации не менее 20 кГц от момента перехода сетевого напряжения через 0 . Момент подачи управляющих импульсов в полупериоде напряжения определяется как разность текущего значения тока диодного плеча и предыдущего отсчета. Когда данная разность становится равной или меньше, чем 0, то в этот момент формируются управляющие импульсы для открытия вступающих в работу плеч ВИП. Блок-схема, реализующая данный алгоритм формирования управляющего импульса $\alpha_{0}$ по предложенному принципу, представлена на рис. 5.

По приходу синхроимпульса запускается алгоритм вычисления изменения тока диодного плеча. Если ток диодного плеча увеличивается (изменение имеет положительный знак), то про-

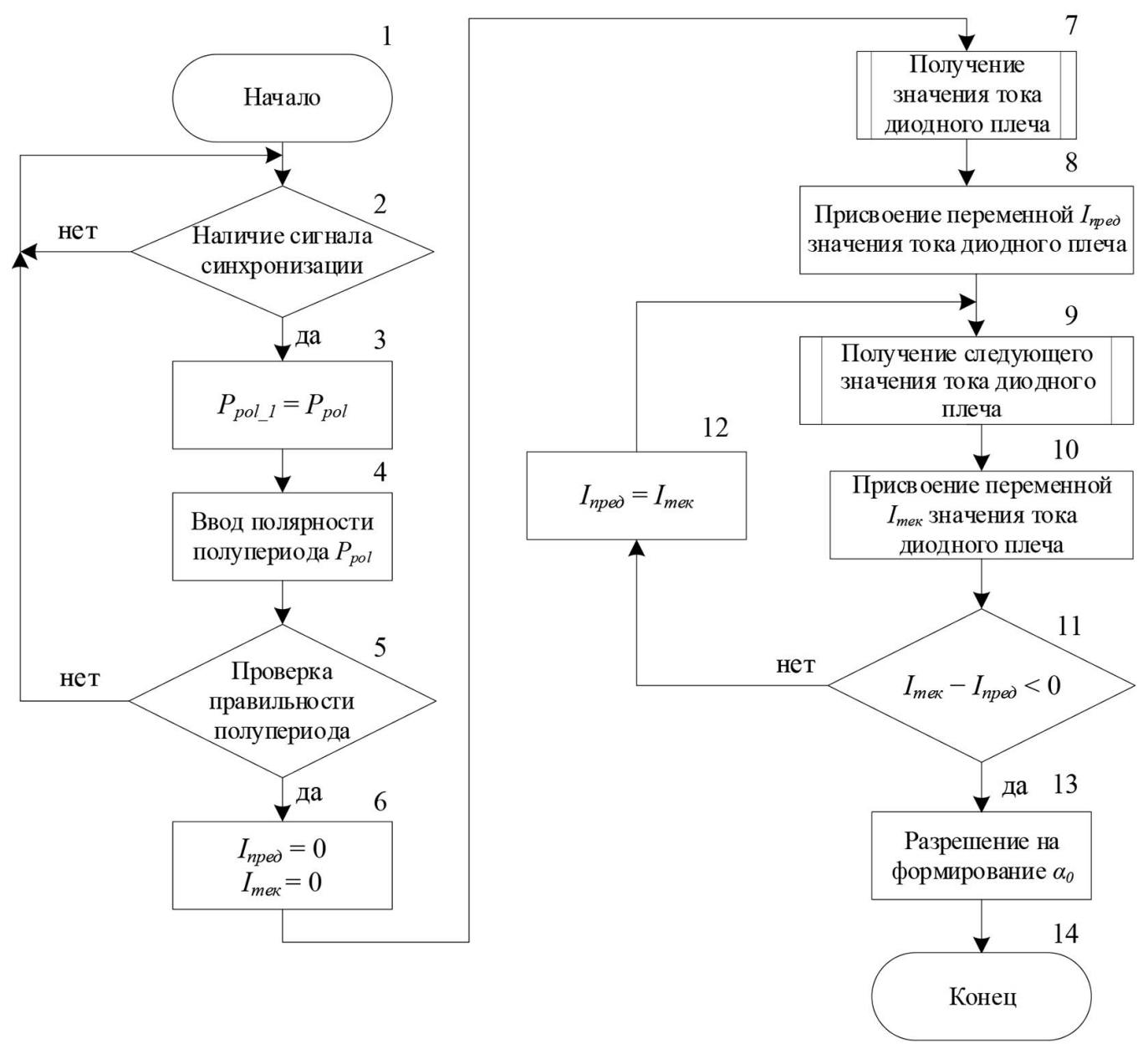

Рис. 5. Алгоритм вычисления момента времени формирования управляющего импульса $\alpha_{0}$ для максимального использования энергии, накопленной в цепи постоянного тока

Fig. 5. Calculating algorithm of control impulse $\alpha_{0}$ point-of-time generation for maximum usage of energy accumulated in the DC subcircuit 
цесс закрытия тиристорных плеч, работавших на предыдущем полупериоде, еще не завершился. Если изменение тока диодного плеча равно нулю или имеет отрицательный знак, то необходимо формировать импульс $\alpha_{0}$ для включения тиристоров плеч преобразователя, образующих контур коммутации, работающий в данном полупериоде напряжения.

Алгоритм реализован следующим образом. В блоке 2 определяется момент перехода сетевого напряжения через ноль. Далее в блоках 3-5 определяется, действительно ли это сигнал смены полупериода или ложное срабатывание из-за искажений сетевого напряжения. Если это действительный переход, то выполняется блок 6, в котором устанавливаются начальные значения переменных текущего и предыдущего значений токов диодного плеча. В блоке 7 производится получение данных от датчика тока диодного плеча. Далее в блоке 8 полученное значение

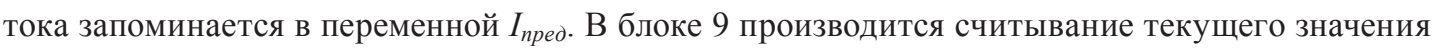

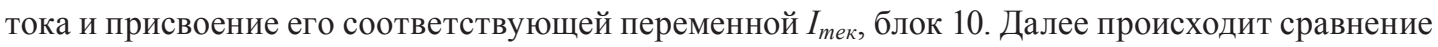
текущего значения тока диодного плеча с предыдущим значением, блок 11, и если разница положительная, то запоминается текущее значение тока, блок 12 , и процесс определения изменения тока диодного плеча повторяется. При разности токов, равной нулю или отрицательной, в блоке 13 формируется сигнал разрешения подачи импульсов $\alpha_{0}$ и работа подпрограммы заканчивается.

\section{Заключение}

1. С целью увеличения коэффициента мощности авторами предлагается новый способ организации сетевой коммутации плеч многозонного выпрямителя электровоза, который использует запасенную в цепи выпрямленного тока электромагнитную энергию для закрытия отработавших тиристорных плеч через контур разрядного диодного плеча, при этом выполняется полезная работа.

2. Предлагаемый способ организации сетевой коммутации плеч многозонного выпрямителя электровоза повышает надежность преобразователя и срок службы полупроводниковых приборов, входящих в его состав, за счет снижения коммутационных токов.

3. Предлагаемый способ организации сетевой коммутации плеч многозонного выпрямителя электровоза реализуется за счет изменения угла открытия тиристоров пропорционально току ТЭД.

4. Предлагаемый новый подход к организации сетевой коммутации плеч многозонного выпрямителя с разрядным диодным плечом повышает коэффициент мощности электровоза в среднем на 3,3 \% относительно штатного.

5. Техническая реализация предлагаемых решений возможна как при модернизации существующих электровозов, так и при выпуске новых и не требует значительных материальных затрат.

\section{Список литературы / References}

[1] Власьевский С. В. Новая организация коммутации тока вентилей выпрямительноинверторного преобразователя для повышения энергетических показателей электровозов переменного тока. Энергосбережение, качество электроэнергии, электромагнитная совместимость на ж.-д. трансп.: сб. тр. ІІ Междунар. симп., М.: РУТ (МИИТ), 2000, 91-93 
[Vlasyevskiy S. V. New Arrangement of Gate Current Commutation of Reversible Power Converter to Increase Energy Performance of AC-Wire DC-Motor Electric Locomotives. Energy Saving, Energy Quality, Electromagnetic Compatibility on Railway Transport: Proc. Anthology of the $2^{\text {nd }}$ Internat. Symp., Moscow, 2000, 91-93 (in Russian)].

[2] Власьевский С. В., Мельниченко О.В. Пути повышения коэффициента мощности электровозов переменного тока с тиристорными преобразователями. Tp. III Междунар. науч. конф. творческой молодёжи, Хабаровск: ДВГУПС, 2003, 1, 68-72 [Vlasyevskiy S.V., Melnichenko O. V. Ways of Increasing Power Factor of AC-Wire DC-Motor Electric Locomotives With Thyristor Converters. Proc. of the $3^{\text {rd }}$ Internat. Sci. Conf. for Creative Youth, Khabarovsk, 2003, 1, 68-72 (in Russian)].

[3] Власьевский С. В., Мельниченко О. В. Применение разрядного диодного плеча в многозонном выпрямителе электровоза переменного тока. Вестн. ВЭлНИИ (Всероссийского науч.исслед. и проектно-конструкторского ин-та электровозостроения), Новочеркасск, 2004, 2, 127-134 [Vlasyevskiy S. V., Melnichenko O. V. Application of a Diode Discharge Arm in a Multizone Rectifier of an AC-Wire DC-Motor Electric Locomotive. Her. of All-Russ. Sci. and Res., Planning and Design Technol. Inst. on Electr. Locomotive Build., Novocherkassk, 2004, 2,127-134 (in Russian)].

[4] Мельниченко О.В., Власьевский С.В. Энергосберегающий алгоритм управления многозонным выпрямителем электровоза с неуправляемым вентиль-диодным плечом. Энергосберегаюшие технологии и окружающая среда: тез. докл. Междунар. конф., Иркутск, 2004, 30-31 [Melnichenko O. V., Vlasyevskiy S. V. Energy-Saving Control Algorithm of an Electric Locomotive Multizone Rectifier With an Uncontrolled Diode Gate Arm. Energy-Saving Technol. and the Environment: Internat. Conf. Rep. Theses, Irkutsk, 2004, 30-31 (in Russian)].

[5] Мельниченко О.В. Повышение энергетической эффективности тяговых электроприводов электровозов переменного тока: дис. ... д-ра техн. наук. Комсомольск-на-Амуре, 2015, 392 c. [Melnichenko O. V. Increasing Energy Efficiency of AC-Wire DC-Motor Electric Locomotive Power Drives: Diss. ... Doctor of Techn. Sci. Komsomolsk-on-Amur, 2015, 392 p. (in Russian)].

[6] Мельниченко О.В., Линьков А. О., Портной А.Ю., Шрамко С.Г. Математическое моделирование работы электровоза с новой выпрямительной установкой возбуждения на IGBT транзисторах. Наука и техника трансn., 2013, 2, 21-28 [Melnichenko O. V., Linkov A. O., Portnoy A. Yu., Shramko S. G. Mathematical Simulation of Electric Locomotive Operation With a New IGBTBased Rectifying Excitation Device. Sci. and Engin. of Transport, 2013, 2, 21-28 (in Russian)].

[7] Мельниченко О.В. Математическое моделирование ВИП электровоза в аварийных режимах с типовым и предлагаемым способами управления. Современные технологии. Сист. анализ. Моделирование, 2013, 4(40), 229-233 [Melnichenko O.V. Mathematical Simulation of Electric Locomotive Reversible Power Converter in Failure Operation Modes With Standard and Proposed Control Methods. Mod. Technol. Syst. Anal. Modelling, 2013, 4(40), 229-233 (in Russian)].

[8] Богинский С.А., Мельниченко О.В. Повышение эффективности использования электромагнитной энергии, запасенной в цепи выпрямленного тока электровоза с целью выполнения полезной работы. Эксплуатация и обслуживание электрон. и микропроцессорного оборудования тягового подвижного состава: сб. тр. Всероссийской науч.-практической конф. с междунар. участием, 2020, 151-159 [Boginskiy S. A., Melnichenko O. V. Increasing Utilization Efficiency of Electromagnetic Energy Stored in Electric Locomotive Power Drive's DC Subcircuit for 
Effective Work. Operation and Maintenance of Traction Rolling Stock Electronic and Microprocessor Equipment: Proc. Anthology of All-Russ. Sci. and Practical conference with international participation, 2020, 151-159 (in Russian)].

[9] Богинский С.А., Мельниченко О.В., Линьков А. О., Шрамко С. Г. Повышение коэффициента мощности электровозов переменного тока за счет новой организации сетевой коммутации плеч выпрямительно-инверторного преобразователя. Современные технологии. Сист. анализ. Моделирование, 2019, 62(2), 166-177 [Boginskiy S. A., Melnichenko O.V., Linkov A.O., Shramko S. G. Increasing Power Factor of AC-Wire DC-Motor Electric Locomotives With Reorganized Grid Commutation of Reversible Power Converter Arms. Mod. Technol. Syst. Anal. Modelling, 2019, 62(2), 166-177 (in Russian)].

[10] Тихменев Б.Н., Басов Ю.А., Находкин В.В. О повышении надежности преобразователя ВИП2-2200М электровоза ВЛ80Р. Вестн. ВНИИЖТ (Всероссийского науч.-исслед. ин-та ж.-д. трансп.), M., 1982, 5, 11-15 [Tikhmenev B. N., Basov Yu. A., Nakhodkin V. V. On Increase of the VL80R Electric Locomotive's VIP2-2200M Converter Series Reliability. Her. of All-Russ. Sci. and Res. Inst. on Railway Transport, Moscow, 1982, 5, 11-15 (in Russian)].

[11] Тихменев Б.Н., Басов Ю. А., Находкин В.В. Потенциальные условия работы тиристоров в выпрямительно-инверторном преобразователе электровоза ВЛ80Р. Электрическое торможение электроподвижного состава: сб. науч. тр., М.: Трансп., 1984, 9-21 [Tikhmenev B.N., Basov Yu. A., Nakhodkin V.V. Potential Conditions of Thyristor Operation in the VL80R Electric Locomotive's Reversible Power Converter. Electr. Braking of Electr. Rolling Stock: Sci. Proc. Anthology, Moscow, 1984, 9-21 (in Russian)].

[12] Корбут Е.А., Рыбин П.Ф. Выбор начальных углов включения тиристорных преобразователей на электроподвижном составе с целью повышения надежности и экономичности. Электровозостроение: сб. науч. тр. ВЭлНИИ, Новочеркасск, 1985, 26, 58-64 [Korbut Е.А., Ribyn P. F. Defining Starting Firing Angles for Electric Rolling Stock Thyristor Converters to Increase Reliability and Cost Effectiveness. Electr. Locomotive Constr.: Sci. Proc. Anthology of All-Russ. Sci. and Res., Planning and Design Technol. Inst. on Electr. Locomotive Build., Novocherkassk, 1985, 26, 58-64 (in Russian)]. 